\title{
SUSTENTABILIDADE E O COMPORTAMENTO DO TURISTA: UMA DISCUSSÃO À LUZ DA PSICOLOGIA AMBIENTAL
}

\author{
SUSTAINABILITY AND TOURIST BEHAVIOR: \\ A DISCUSSION IN THE LIGHT \\ OF ENVIRONMENTAL PSYCHOLOGY
}

Data de submissão: 08-12-2014 Aceite: 01-08-2015

Cristiane Salome Ribeiro Costa ${ }^{1}$ Jaqueline Guimarães Santos ${ }^{2}$ Edvan Cruz Aguiar ${ }^{3}$

\section{RESUMO}

O estudo sobre o comportamento do turista tem despertado crescente interesse nas áreas de comportamento do consumidor e sustentabilidade, uma vez que é visto como um agente que influencia diretamente a sustentabilidade do destino turístico. Sob a ótica da Psicologia Ambiental, mais especificamente do modelo S-O-R de Mehrabian e Russell (1974), entende-se que o turista pode ser influenciado por estímulos existentes no ambiente, já que estes, representados pelas características e demais informações concernentes às dimensões da sustentabilidade, dispostos na destinação turística, correspondem a variáveis que podem exercer influência sobre o comportamento do turista. Nesse sentido, o presente ensaio propôs-se a discutir a relação entre as características de sustentabilidade de destinos turísticos e o comportamento do turista. Acredita-se que o destino turístico é capaz de estimular comportamentos voltados para a sustentabilidade, desde que consiga utilizar adequadamente as características do ambiente para informar e instruir o turista, sobretudo aquelas relacionadas às dimensões da sustentabilidade. Este trabaIho também propõe um framework na tentativa de melhor compreender a relação aqui discutida e sugerir estudos empíricos posteriores que compartilhem do mesmo intento.

Palavras chave: Comportamento do Turista; Psicologia Ambiental; Sustentabilidade; Destinos Turísticos; Modelo S-O-R.

1 Possui graduação em Administração pela Universidade Federal de Pernambuco - UFPE, mestrado em Administração pela Universidade Federal de Pernambuco - UFPE, doutorado em Master in Imagineering. NHTV Breda University of Applied Sciences, NHTV, Holanda e doutorado em andamento em Administração pela Universidade Federal de Pernambuco - UFPE. Atualmente é professor efetivo da Universidade Federal de Pernambuco - UFPE. Recife. Pernambuco. Brasil. E-mail: csrcosta@yahoo.com.br

2 Possui graduação interrompida em 2009 em Ciências Biológicas pela Universidade Estadual da Paraíba - UEPB, graduação em Administração pela Universidade Federal de Campina Grande - UFCG e mestrado em Administração pela Universidade Federal de Pernambuco - UFPE. Atualmente é professora Assistente da Universidade Federal de Pernambuco - UFPE. Caruaru. Pernambuco. Brasil. E-mail: jsantos.adm@gmail.com

3 Possui graduação em Bacharelado em Administração pela Universidade Estadual da Paraíba - UEPB, mestrado em Administração pela Universidade Federal de Pernambuco - UFPE e doutorado em andamento em Administração. Universidade Federal de Pernambuco - UFPE. Atualmente desenvolve pesquisas nas áreas de Comportamento do Consumidor, Varejo, Marketing de Serviços, Ensino e Pesquisa em Administração. Recife. Pernambuco. Brasil. E-mail: edvan.ed@gmail.com 


\section{ABSTRACT}

The study on tourist behavior has aroused increasing interest in the areas of consumer behavior and sustainability, since this is seen as an agent that directly influences the sustainability of the destination. In light of environmental psychology, specifically the SOR of Mehrabian and Russell (1974) model, it is understood that the tourist can be influenced by stimuli in the environment. Apprehends that environmental stimuli, represented by features and other information concerning the dimensions of sustainability, arranged in a tourist destination, corresponding to the variables that can influence the behavior of tourists. In this sense, this essay proposes to discuss the relationship between the characteristics of sustainable tourism destinations and tourist behavior. It is believed that the tourist destination can stimulate behaviors towards sustainability, provided that it can properly utilize the characteristics of the environment to inform and educate tourists, especially those related to the dimensions of sustainability. The paper also proposes a framework in an attempt to better understand the relationship discussed and subsequent empirical studies suggest that share the same intent.

Keywords: Behavior of Tourist; Environmental Psychology; Sustainability; Tourist Destinations; Model S-O-R.

\section{INTRODUÇÃO}

Os estudos acerca da sustentabilidade vêm se tornando cada vez mais frequentes no campo acadêmico, em suas diversas áreas (VAN DER ZWAN; BHAMRA, 2003), sobretudo no campo do turismo (KOROSSY, 2008). Desde meados da década de 90, a questão ambiental tem exercido influência nas pesquisas nesta área de conhecimento (KOROSSY, 2008), em função do rápido crescimento das atividades turísticas. Por outro lado, essa atividade econômica tem acarretado danos à biodiversidade e às características das localidades visitadas (MARTINS, 2002; CANDREA, ISPAS, 2009; OMT, 2010), causando a exploração excessiva do espaço natural e a interferência nas identidades culturais das destinações turísticas (ELIGH, WELFORD, YTTERHUS, 2002; JAMROZY, 2007).

Derivado da terminologia da sustentabilidade, a perspectiva do turismo sustentável desenvolve-se objetivando direcionar as ações turísticas para seguir os critérios de relevância social, prudência ecológica e viabilidade econômica, como preza a abordagem sustentável, visto que os ambientes que constituem a base da atividade compreendem aspectos de cunho ambiental, cultural e econômiCo (KOROSSY, 2008). Sendo assim, a definição que mais se aproxima desse escopo é apresentada pela Organização Mundial do Turismo (OMT, 2010), conceituando o turismo sustentável como qualquer atividade preocupada em gerenciar todos os recursos que caracterizam sua oferta, de tal forma que necessidades econômicas, sociais e estéticas sejam atendidas mantendo a integridade cultural, os processos essenciais ecológicos, a biodiversidade e os sistemas de suporte à vida.

Ressalta-se que, apesar de essa definição ser aceita e referenciada na literatura (KOROSSY, 2008; CANDREA, ISPAS, 2009; ALONSO, OGLE, 2010), é comum que os destinos turísticos não explorem a contento todas as dimensões constitutivas do conceito de sustentabilidade. $\mathrm{Na}$ maioria dos casos, concentram-se em apenas uma das dimensões que compõem o tripé da sustentabilidade: econômica, social ou ambiental.

Uma das consequências desse foco restrito em torno de um dos critérios definidores da sustentabilidade é o tipo de direcionamento que pode provocar sobre o comportamento do turista. De acordo com a abordagem do comportamento sustentável, os papéis que os indivíduos desempenham com relação à sustentabilidade em destinações turísticas irão variar dependendo das situações e do contexto (GALLARZA; GARCIA; SAURA, 2002). Isso implica afirmar que, dependendo do tipo de característica sustentável explorada pela destinação turística, os turistas tenderão a desenvolver comportamentos alinhados a dimensões presentes no ambiente ao seu 
redor (MCDONALD et al., 2009; PREBENSEN, FOSS, 2011). Desse modo, se o ambiente no qual o turista está envolvido enfatiza uma dimensão da sustentabilidade em detrimento das demais, ele tenderá a se comportar de acordo com tais indicativos.

A Psicologia Ambiental, enquanto área de conhecimento envolvida no estudo e entendimento das relações entre indivíduos e seus contextos ambientais, pode explicar a ocorrência da relação supracitada. Especificamente o modelo estímulo $(\mathrm{S})$, organismo $(\mathrm{O})$ e resposta $(\mathrm{R})$, com origem na abordagem de Mehrabian e Russell (1974), trabalha sob a lógica da relação entre o estímulo que o ambiente empreende sobre o sujeito e o poder de influenciá-lo em suas atitudes e seus comportamentos.

$\mathrm{O}$ organismo $(\mathrm{O})$ percebe, organiza e interpreta as informações $(\mathrm{S})$, gerando respostas $(\mathrm{R})$ como consequência dessa relação, que resulta no seu comportamento (GIULIANI, 2005). Os estímulos podem originar-se tanto na estrutura do ambiente (aspectos físicos, por exemplo) quanto na influência das pessoas presentes na destinação, especialmente quando são importantes fontes de referência pela falta de conhecimento do turista em relação à atividade (PINHEIRO, 1997; GIULIANI, 2005).

Diante do exposto, a partir da Psicologia Ambiental, especificamente da abordagem S-O-R de Mehrabian e Russell (1974), o presente ensaio tem como objetivo discutir a relação entre as características sustentáveis de destinos turísticos e o comportamento do turista. A argumentação aqui apresentada parte do pressuposto de que as informações (estímulos) presentes na destinação, percebidas como representação das dimensões da sustentabilidade, são variáveis que interferem no comportamento sustentável do turista ao experienciar os destinos turísticos (GREAVES, ZIBARRAS, STRIDE, 2013; MEHRABIAN, RUSSELL, 1974; GIULIANI, 2005).

\section{SUSTENTABILIDADE: UMA VISÃO MACRO}

Com seu início aproximadamente em 1972, as pesquisas sobre a sustentabilidade permeiam as investigações nas mais diversas áreas, com o principal intuito de identificar as variáveis que podem contribuir para o seu alcance (CERNAT; GOURDON, 2007). Inicialmente definida como "capacidade de um modelo ou sistema sustentar-se na dinâmica evolutiva sem permitir que algum setor se aprofunde em crises de tal forma que venha a atingir a totalidade" (BRUNDTLAND, 1987 apud RUSCHEINSKY, 2003, p. 5), a abordagem da sustentabilidade atualmente apresenta uma perspectiva mais voltada para a inclusão de questões relacionadas à qualidade de vida e ao desenvolvimento social, ambiental e econômico.

De modo geral, as discussões acerca da sustentabilidade vêm se fundamentando sobre o alcance do equilíbrio entre as dimensões social, econômica e ambiental, intituladas de tripé da sustentabilidade (ELKINGTON, 1999). No entanto, a falta de critério ou padrão em torno do status e do nível da sustentabilidade tem gerado diferentes formatos de implementação e monitoração das suas dimensões $(K O, 2005)$. Em função da complexidade inerente ao tema, cada área de investigação aborda a temática de acordo com determinada base epistemológica, aproximando a aplicação dos conceitos sustentáveis ao seu contexto.

Dessa forma, os critérios da sustentabilidade podem não ser desenvolvidos seguindo os mesmos parâmetros. Utilizar inovações na produção ou no redesenho dos produtos/serviços com o intuito de diminuir o uso dos recursos pode representar o foco de apenas uma das dimensões da sustentabilidade (ALIGLERI; ALIGLERI; KRUGLIANSKAS, 2009). Da mesma forma, o trabalho do ecodesign para reduzir os impactos de um produto no ambiente (KAZAZIAN, 2005; LAKHANI, 2007) ou um esforço desenvolvido em prol da comunidade que promova o crescimento social e a qualidade de vida, por exemplo, pode ser construído, assim, com base em apenas uma das dimensões supracitadas (SACHS, 2007; PRAHALAD, 2005). 
No entanto, essas atribuições da sustentabilidade de acordo com o contexto são suscetíveis a críticas. Uma dessas críticas foi realizada por Fergus e Rowney (2005), que consideram importante analisar com cautela a construção de atividades, levando em consideração pesos diferentes sobre os critérios da sustentabilidade, a fim de não comprometer o seu alcance. Já Buarque (2008) entende que o foco em apenas uma das dimensões da sustentabilidade está mais próximo de um discurso que do desenvolvimento prático da sustentabilidade, indicando a tentativa de promover um aparente interesse ambiental e social. Isso implica que, dentro das organizações, é possível utilizar-se de uma linguagem sem nenhum significado real, ou seja, sem que o discurso seja implementado na prática.

$\mathrm{Na}$ verdade, essas críticas chamam atenção para o cuidado na aplicação do conceito de sustentabilidade, a fim de evitar uma visão utilitarista baseada essencialmente na busca do ganho econômico (GADENNE; KENNEDY; MCKEIVER, 2009). A preocupação de Fergus e Rowney (2005) e Buarque (2008) ganha corpo em trabalhos como de Porter e Van der Linde (2003), que retratam o desenvolvimento da sustentabilidade como estratégia para obter vantagem competitiva, e de Orsato (2002), que propõe a adequação da estrutura e do desempenho organizacional aos pressupostos da sustentabilidade, com o intuito de ajudar a aumentar o número de clientes e a lucratividade. Além do aspecto estratégico, a visão utilitarista também recai na perspectiva do nicho de mercado. Pressupõe-se que empresas podem ofertar produtos e serviços sustentáveis a um grupo específico de consumidores, que possuem consciência sobre a importância da sustentabilidade, para ganhar uma imagem positiva na sociedade (MARIN; RUIZ, RUBIO, 2009; KOTLER, KARTAJAYA, SETIAWAN, 2010).

Todavia, em detrimento de críticas em torno das vantagens de alcançar lucratividade diante da ênfase em alguma das variáveis sustentáveis, entende-se que o êxito da sustentabilidade pode ser encontrado na aplicação efetiva de algumas de suas dimensões, mesmo que isoladamente. Frente à complexidade em designar quais atividades podem contemplar todas as dimensões da sustentabilidade de forma equilibrada e, até mesmo, em identificar quais delas, de fato, podem ter um impacto positivo, propor a aplicação de alguns dos critérios sustentáveis permite que ocorra um aprimoramento contínuo, já que as proposições saem do campo das ideias e passam a ser analisadas na prática (HARRIS, 2000). Isso traz uma contribuição do ponto de vista teórico à medida que as ações reais em torno da sustentabilidade se tornam observadas e avaliadas, permitindo que outras perspectivas possam ser vislumbradas (HART, 2006; HARRIS, 2000; FOLADORI, 2005).

Além disso, como a sustentabilidade pode ser trabalhada em múltiplos níveis, sendo capaz de ser alcançada somente se os subsistemas interdependentes forem viáveis e sustentáveis (HART, 2006), analisar isoladamente a aplicação das variáveis permite identificar de forma mais clara como essas conexões podem ser produzidas e avaliar quais atividades da interação merecem atenção para conduzir a sustentabilidade em outras dimensões (NASCIMENTO, 2005; MACHADO FILHO, 2006), especialmente no tocante ao papel do consumidor em determinados contextos que possuem um impacto maior em determinados critérios em torno da sustentabilidade, como é o caso do ambiente turístico.

No campo do turismo sustentável, o qual deriva do próprio conceito de sustentabilidade, a discussão entre as dimensões que o compõem e as que são realizadas na prática também prevalecem. A seção seguinte aborda justamente o posicionamento sobre esta relação. 


\section{TURISMO SUSTENTÁVEL}

Desde meados das décadas de 80 e 90, a questão ambiental tem exercido influência sobre os estudos na área turística (KOROSSY, 2008). De acordo com Eligh, Welford e Ytterhus (2002), o rápido crescimento da atividade tem gerado altos índices de problemas relacionados à poluição, impactos na biodiversidade, crescimento econômico desordenado, especulação imobiliária, degradação ambiental, modificação das fontes culturais por meio da introdução de elementos estrangeiros etc. (JAMROZY, 2007). A propagação do turismo de massa vem aprofundando esse debate, tendo em vista a fragilidade da comunidade e dos recursos (HAZIN; OLIVEIRA; MEDEIROS, 2001).

O conceito de turismo sustentável é derivado de desenvolvimento sustentável, mas foi apenas em 1992, no evento "Agenda 21", que o turismo passou a fazer parte da pauta de discussões, a partir de um capítulo chamado de Carta do Turismo Sustentável (Charter for Sustainable Tourism) (UNWTO, 2004). O documento chamava a atenção para o fato de que:

O desenvolvimento da atividade turística não deve ultrapassar os limites do ambiente natural, deve ser economicamente viável e equânime para as comunidades locais; deve haver a participação dos atores sociais envolvidos nos níveis local, regional, nacional e internacional; o planejamento do turismo deve ser elaborado por governos e autoridades competentes, contando com a participação das comunidades locais e de organizações não governamentais, de forma integrada; defende a adoção de códigos de conduta; promoção de formas alternativas de turismo (KOROSSY, 2008, p. 62).

Sendo assim, as prerrogativas da sustentabilidade devem ser aplicadas também ao turismo, de modo que os aspectos social, cultural e ecológico se tornem tão importantes quanto os ganhos econômicos e atentando aos ambientes que constituem a base da atividade turística - o natural, cultural e patrimonial (CURTIN; BUSBY, 1999). Para a Organização Mundial do Turismo (OMT, 2003), o turismo é responsável por administrar todos os recursos de tal forma que necessidades econômicas, sociais e estéticas possam ser contempladas enquanto se mantêm a integridade cultural, os processos essenciais ecológicos, a biodiversidade e os sistemas de suporte à vida. Cernat e Gourdon (2007), por sua vez, entendem que o turismo, por contemplar um conjunto de atividades em contínuo crescimento econômico, deve alcançar as metas de sustentabilidade com relação ao meio ambiente, ao sistema social e à identidade cultural.

A amplitude da definição da sustentabilidade abre espaço para diferentes interpretações, o que representa na prática dificuldades para operacionalizar o turismo sustentável. Essas questões têm se tornando presentes em um complexo debate sobre como utilizar os recursos naturais a fim de criar, manter e ampliar o bem-estar humano e, ao mesmo tempo, preservá-los, bem como de promover a distribuição de custos e benefícios (BOB; GHITA; SASEANU, 2010).

Diante da complexidade que envolve o tema, é possível observar que as ações protagonizadas sobre o turismo sustentável podem não alcançar o equilíbrio entre os critérios de relevância social, prudência ecológica e viabilidade econômica, conforme rege o princípio da sustentabilidade. Como resultado, torna-se comum que os estudos analisem as atividades em termos de uma das variáveis, usando metodologias que não englobam a amplitude dos aspectos relacionados à temática no turismo (MOSCARDO, 2007). Embora esteja estritamente associado a questões ambientais, podem-se encontrar várias interpretações desse conceito (KOROSSY, 2008), como, por exemplo, turismo rural e ecoturismo, para a dimensão ambiental; turismo cultural e patrimonial, para a dimensão social; e o desenvolvimento urbano, para a dimensão econômica (GÖSSLING et al., 2002).

Já para Coccossis (1996), Hall (2000) e Towar e Lockwood (2008), essas aproximações não poderiam ser vistas como turismo sustentável, porque, de uma forma ou de outra, pro- 
vocam consequências negativas em alguma das dimensões da sustentabilidade. Hunter (1997), Ko (2005) e Santos (2013), por sua vez, argumentam que essa aproximação é possível, pois as dimensões e os interesses relacionados ao turismo sustentável podem variar de uma destinação para outra e há uma necessidade de tais dimensões serem trabalhadas de forma conjunta e equilibrada em qualquer que seja o destino turístico. Esses autores sugerem que, embora as dimensões, os indicadores e os dados possam variar para adaptar a metodologia às especificidades de cada destinação turística, tais dimensões precisam ser exploradas de forma concomitante para que se alcance o turismo sustentável.

No entanto, observa-se que os destinos turísticos não planejam a atividade turística buscando o equilíbrio e a equidade entre as dimensões. As atividades turísticas baseadas no critério ambiental, por exemplo, enfatizam ações voltadas para a preservação do meio ambiente, como estabelecer limite ao número de visitantes para áreas protegidas e incentivar a diminuição dos resíduos e dos gastos dos recursos naturais (CERNAT, GOURDON, 2007; CLAYTON, 2009; GRAČAN, SANDER, RUDANČIĆ-LUGARIĆ, 2010). Já as atividades turísticas que têm como identidade a cultura ou o patrimônio tendem a focalizar a valorização da comunidade e da interação desta com o visitante ou, ainda, a preservação das características tanto culturais quanto patrimoniais da localidade (DONYADIDE, 2005; RICHINS, 2009; ALONSO, OGLE, 2010). Investimentos em infraestrutura, circulação de renda, incremento na oferta de empregos e qualificação da mão de obra da população local foram, por sua vez, alguns dos resultados relacionados à valorização do aspecto econômico (MARTINS, 2002; LE, 2005).

É importante, contudo, que as atividades turísticas considerarem, desde o planejamento da atividade até sua execução, as dimensões ambiental, social e econômica, de modo que se tenha um turismo sustentável que contribua para o desenvolvimento local sustentável. Nesse sentido, apreende-se a pertinência de pensar não apenas nos aspectos econômicos, mas também nos fatores sociais e ambientais, que devem ser considerados em iguais proporções (MOSCARDO, 2007).

Sabe-se que a caracterização de uma destinação pode ter influência sobre o comportamento sustentável do turista, de modo que, quando o enfoque de tal atividade é apenas em uma dimensão, o comportamento do visitante tende para a dimensão na qual a localidade enfoca suas ações. A seção seguinte aborda a perspectiva da Psicologia Ambiental sobre o tema, discorrendo acerca da influência que o ambiente externo tem sobre o comportamento dos indivíduos.

\section{PSICOLOGIA AMBIENTAL}

O primeiro autor a utilizar o termo Psicologia Ambiental foi Brunswik, em 1943 (GIFFORD, 2002). A Psicologia Ambiental é uma subárea da Psicologia, que tem como objeto de estudo as inter-relações entre as pessoas e suas ações com o meio ambiente (ALVES; BASSANI, 2008).

De acordo com Bassani (2010), a Psicologia Ambiental firma-se no meio científico na década de 70, com a proposta de realizar investigações no contexto das relações entre os seres humanos, os ambientes físicos e os problemas ambientais, buscando novas formas de atuação e produção do conhecimento. Desse modo, a ênfase encontra-se no estudo da inter-relação pessoa-ambiente, entendendo que as pessoas alteram o ambiente e este também interfere no comportamento humano.

A definição de Moser (2005), de que a Psicologia Ambiental estuda a pessoa em seu contexto, tendo como tema central as inter-relações - e não somente as relações - entre a pessoa e o ambiente físico e social, faz surgir diversas questões a respeito da maneira como essas inter-relações são abordadas. Por isso, na história dessa área da Psicologia, é possível identificar diversas vertentes. De acordo com Freire e Vieira (2006), a multiplicidade de perspectivas também 
repercute nos diálogos interdisciplinares que a Psicologia Ambiental estabelece, principalmente com a arquitetura, a geografia, a ecologia, as ciências sociais e as diversas áreas da Psicologia, como a social, a comunitária e, até mesmo, a comportamentalista. Logo, entende-se que sua atuação assume caráter interdisciplinar e transdisciplinar e que a Psicologia precisa se engajar na análise e no encaminhamento de soluções para os problemas ambientais, pois estes são, de fato, problemas humano-ambientais (PINHEIRO, 1997).

Em face disso, o argumento presente neste ensaio fundamenta-se na perspectiva do mecanismo estímulo-organismo-resposta (S-O-R), o qual deu início aos estudos que relacionam o ambiente ao comportamento de consumo (MEHRABIAN; RUSSELL, 1974). Para estes estudiosos, as respostas aos estímulos de um ambiente são causadoras de comportamentos de aproximação ou afastamento. Além disso, esses estímulos ambientais, quando bem-utilizados, trariam às pessoas mais disposição ou desejo de ir ao ambiente, permanecer nele ou explorá-lo, interferindo, assim, no comportamento do consumidor.

No âmbito do consumo turístico, quanto à relação do turista com a destinação, mais especificamente em termos de comportamento, entende-se ser pertinente uma discussão sobre como os estímulos físicos dispostos na localidade podem influenciar o indivíduo a ter um comportamento sustentável. Ademais, tal discussão versaria sobre o comportamento sustentável do turista e o conceito de consumidor sustentável, bem como delinearia características e estratégias pertinentes a serem desenvolvidas para direcioná-lo a um comportamento sustentável. De acordo com Pinheiro (1997), apesar de importantes aproximações entre disciplinas voltadas para a questão ambiental (por exemplo, Ecologia e Economia), faz-se necessário um maior esforço para compreender o ser humano e sua relação com o ambiente.

Diante disso, entende-se que esta perspectiva oferece oportunidade para o desenvolvimento teórico e científico sobre a forma de agir dos turistas, enquanto consumidores que exploram, vivenciam e influenciam o ambiente. Ademais, pode auxiliar na proposição de ações que caminhem rumo a um comportamento de consumo sustentável. A Figura 1, exposta a seguir, apresenta o modelo S-O-R (estímulo-organismo-resposta) desenvolvido por Mehrabian e Russel (1974).

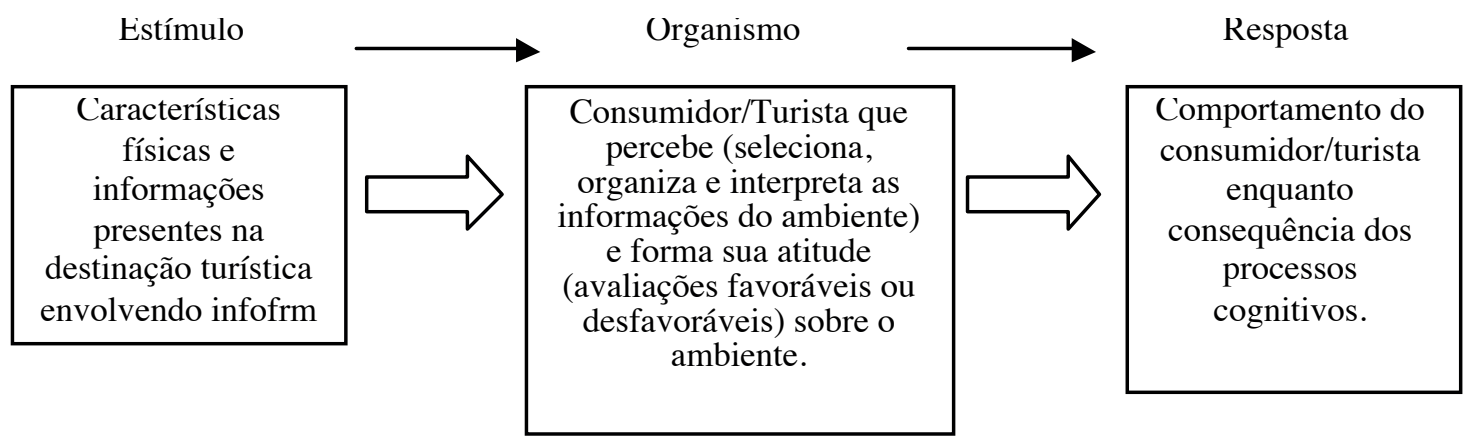

Figura 1: Sistemática do comportamento do turista.

Fonte: adaptado de Mehrabian e Russell (1974).

Acredita-se que o comportamento do turista seja fortemente influenciado pelas informações e características presentes na destinação turística, de forma que a maneira como esses estímulos são empreendidos sobre o turista tenderá a direcionar suas ações segundo os indicativos presentes no ambiente visitado. Esses estímulos podem vir da composição do ambiente, incluindo características físicas que promovem o direcionamento sobre o comportamento do turista (STEG; VLEK, 2009), e, também, dos próprios indivíduos que frequentam e compõem o local (PAYNE et al., 2009). Portanto, 
as próprias destinações podem direcionar o comportamento dos turistas conforme sua oferta de experiência, sobretudo objetivando a não interferência na sustentabilidade do destino.

Nesse sentido, é possível estabelecer alguns determinantes do comportamento do turista, que correspondem a fatores internos e externos a ele. A destinação turística em si, as informações dispostas na localidade (folhetos, folders etc.) e as outras pessoas presentes corresponderiam a elementos externos. Já os aspectos cognitivos, especialmente percepção, personalidade, motivação e atitude, dizem respeito aos aspectos intrínsecos. Na seção seguinte, são discutidos alguns aspectos que podem influenciar o comportamento do turista.

\section{O COMPORTAMENTO DO TURISTA A PARTIR DO EN- FOQUE DADO PELO DESTINO TURÍSTICO}

Em virtude do alto fluxo de atividades turísticas, que envolvem visitas a lugares motivadas pela natureza, cultura e religião, por exemplo, entendendo que o indivíduo pode impactar negativamente a sustentabilidade de uma localidade, uma das investigações do turismo sustentável está relacionada ao comportamento do turista (CANDREA; ISPAS, 2009). Para que, de fato, o turismo sustentável possa ser alcançado, os turistas devem direcionar suas ações de consumo prezando os valores da sustentabilidade (HUNT, 2011). Nesse sentido, a investigação em torno do comportamento do turista concentra-se no entendimento das suas características e das estratégias a serem desenvolvidas a fim de direcioná-lo a um comportamento sustentável. De uma forma geral, o turista ainda não tem o seu papel claramente definido, principalmente no que diz respeito à sua participação nas ações envolvendo a sustentabilidade e o consumo sustentável.

Para Castaldo et al. (2009), o consumidor é visto apenas como o receptor das ações oriundas das empresas e do governo, enquanto que Aligleri, Aligleri e Kruglianskas (2009) entendem que o consumidor, por ser o elo final da cadeia produtiva, não ocasiona tantos danos quanto a indústria do setor, motivo pelo qual acaba sendo considerado um fator secundário para os estudos.

O seu papel tambem é considerado contraditório, porque, se, de um lado, há uma tendência positiva de consciência do consumidor sobre as questões da sustentabilidade (KOTLER; KARTAJAYA; SETIAWAN, 2010), de outro lado, este pouco ou nada faz para mudar seus próprios comportamentos (POMERING; NOBLE; JOHNSON, 2011). No entanto, como entendem Kotler, Kartajaya e Setiawan (2010), são perceptíveis algumas mudanças de comportamentos nos consumidores, já que, por estarem mais conscientes do que representa a sustentabilidade, tornam-se mais exigentes e cobram dos governos e das empresas produtos e serviços mais sustentáveis. Isso significa que, se as empresas não desenvolverem produtos ou serviços com esse foco, não irão proporcionar a identificação com o consumidor, o qual buscará outras empresas que possam corresponder aos seus anseios por itens sustentáveis (MARIN; RUIZ; RUBIO, 2009). Apesar disso, na prática, muitos consumidores não fazem distinção entre consumir um produto/serviço de uma empresa que invista em sustentabilidade e consumir de outra que não tenha um apelo mais sustentável (CASTALDO et al., 2009; POMERING, NOBLE, JOHNSON, 2011).

Esse paradoxo pode explicitar a lacuna existente entre o discurso e a prática do consumidor (DAWKINS, 2004). Liu (2003) observa que a diferença entre a consciência do consumidor e suas ações acerca da sustentabilidade pode ser explicada com base no fato de que, para os consumidores, a obrigação de desenvolver atividades mais sustentáveis é das empresas e do governo, de modo que seu comportamento sustentável só ocorrerá a partir de tais obrigações impostas. É possivel que, se obrigado, o consumidor se comporte de forma diferente, caso não tenha outra opção de escolha (LIU, 2003). 
Phipps et al. (2012) chamam atenção, entretanto, sobre a forma como as pesquisas sobre o tema são desenvolvidas. Partem do pressuposto de que os consumidores são racionais e agem sistematicamente de acordo com seus valores, de modo que suas escolhas se baseiam em informações acerca da sustentabilidade do produto ou serviço. Muitas fazem uso de metodologias que consideram o consumidor sendo afetado pelos mesmos fatores em diferentes contextos de compra. O que efetivamente ocorre é que existem fatores influenciadores que podem direcionar o comportamento em prol da sustentabilidade e que devem ser analisados sob a perspectiva de cada situação de consumo em separado. Isso leva a inferir que o comportamento sustentável não é nem consistente nem coerente e que um mesmo indivíduo pode desempenhar diferentes papéis considerando o contexto e a situação em que está inserido (MCDONALD et al., 2009).

Seus valores não são expressos da mesma maneira, e o ambiente, de certa forma, pode direcionar o consumidor a um determinado comportamento (PEATTIE, COLLINS, 2009; ROKKA, MOISANDER, 2009; PHIPPS et al., 2012). Assim como Liu (2003) afirma que o comportamento do consumidor sustentável depende em grande parte da infraestrutura (tratada pelo autor como obrigação de governos e empresas), McDonald et al. (2009) entendem que, de fato, o ambiente externo ao indivíduo exerce uma importante influência não apenas do ponto de vista do comportamento, mas também com relação à atitude que corresponde a um indicativo de ação. O que é perceptível aos olhos do turista pode ser um fator para direcionar o seu comportamento para que proceda alinhado aos princípios de sustentabilidade no âmbito da atividade turística.

McDonald et al. (2009) e Rokka e Moisander (2009) atentam para o fato de que a sustentabilidade, muitas vezes, é percebida como um critério utilizado para a tomada de decisão nas destinações turísticas, assim como preço, tempo, habilidade e conhecimento. Nesse sentido, Molina (2001) afirma que os turistas atualmente estão interessados na qualidade ambiental dos lugares que visitam, o que condiciona sua decisão de visitá-los ou não. $O$ autor apresenta uma pesquisa feita com turistas alemães, em que estes enumeraram as seguintes prioridades ao escolher um destino para as férias: praias limpas, baixo nível de ruído, baixo nível de densidade na ocupação do espaço, higiene, dentre outros fatores.

A partir da Psicologia Ambiental, especialmente do modelo S-O-R (estímulo-organismo-resposta), é coerente afirmar que o turista é diretamente influenciado pelo ambiente, de forma que as dimensões da sustentabilidade presentes na destinação turística direcionarão o comportamento do indivíduo conforme os aspectos/estímulos presentes no destino turístico. Isso significa que, caso o ambiente construa a sua base voltada ao meio ambiente, o turista tenderá a desenvolver um comportamento voltado para a preservação do ambiente. Desse modo, à luz do modelo $\mathrm{S}-\mathrm{O}-\mathrm{R}$, as variáveis ambientais em destaque podem ser entendidas como variáveis mediadoras do comportamento do turista em ambientes turísticos sustentáveis.

Turismo rural, ecoturismo e turismo de área preservada tenderão a desenvolver no indivíduo uma consciência sobre a preservação dos recursos naturais, direcionando seu o comportamento para a proteção ao meio ambiente, o recolhimento do próprio lixo e o uso dos recursos naturais. Assim também ocorrerá com os outros critérios da sustentabilidade, tanto o social quanto o econômico. Para atividades turísticas que prezam pela relevância social, especialmente o turismo cultural e patrimonial, os estímulos presentes no ambiente serão voltados para a valorização do entorno, conduzindo os turistas para a participação em atividades culturais, a hospedagem na residência dos nativos e a participação na produção de artesanatos (dimensão social). Já quando o destaque for destinado ao critério econômico, com ações do processo turístico voltadas para melhorias na infraestrutura e circulação de renda (dentre outros), o consumidor poderá centrar suas atitudes na relação de custo-benefício. 
Os estímulos, então, podem ser apresentados a partir de informações dispostas no ambiente de visitação. Essas informações podem ser exibidas em sites de busca de dados, em sites oficiais e no próprio local de visita, por meio de folhetos e folders informativos.

A partir do momento em que o turista recebe a orientação sobre as consequências de uma ação voltada para a sustentabilidade, entende-se que esta será trabalhada e praticada pelo visitante (MCNEILL; VAUGHN, 2012). Essa afirmação é oriunda de um estudo conduzido por Jakobsson, Makitalo e Saljo (2009), que revelou que o conhecimento sobre as consequências do aquecimento global por parte de um grupo de estudantes melhorou o seu comportamento com relação à sustentabilidade na destinação, gerando participação sobre as ações desenvolvidas, além do sentimento de responsabilidade e cuidado para com a natureza.

Assim, entende-se que é possível influenciar o comportamento do turista a partir das características do destino turístico, o que poderá moldar o seu comportamento em direção à sustentabilidade (XIANG; PETRICK, 2009). Torna-se importante observar, também, que tal comportamento pode ser moderado pelas características intrínsecas do consumidor. Conforme Phipps et al. (2012) propõem, os consumidores comportam-se de forma sustentável mediante a orientação de valor que possuem, considerando as variáveis que a compõe: hábitos e conhecimento, condições situacionais (tempo, dinheiro e capacidade cognitiva) e habilidades. Essa observação leva ao entendimento de que os turistas com perfis ou tendências mais sustentáveis, além do próprio conhecimento, poderão sofrer uma influência maior dos critérios da sustentabilidade presentes no destino do que aqueles que não os possuem.

Isso não implica afirmar que o ambiente não exerce influência sobre o comportamento do indivíduo. Conforme apontam Prebesen e Foss (2011), estando no destino, o turista tenderá a responder aos estímulos por motivos cognitivos ou afetivos como estratégia para maximizar os benefícios centrais esperados e para evitar problemas de afastamento ou exclusão por parte de outros componentes do grupo e dos anfitriões. Na realidade, dependendo da orientação de valor do turista sobre a sustentabilidade, a intensidade pode ser maior naqueles que apresentem uma predisposição elevada para responder aos seus estímulos, mas, ainda assim, os que possuem uma baixa ou moderada predisposição, de alguma forma, corresponderão às demandas ambientais, seja por características do destino ou pelo comportamento dos outros turistas.

Nesse sentido, a partir desses fatores, elaborou-se um framework para melhor compreender as relações entre o comportamento do turista e o enfoque dado pelo destino turístico, conforme apresentado na Figura 2.

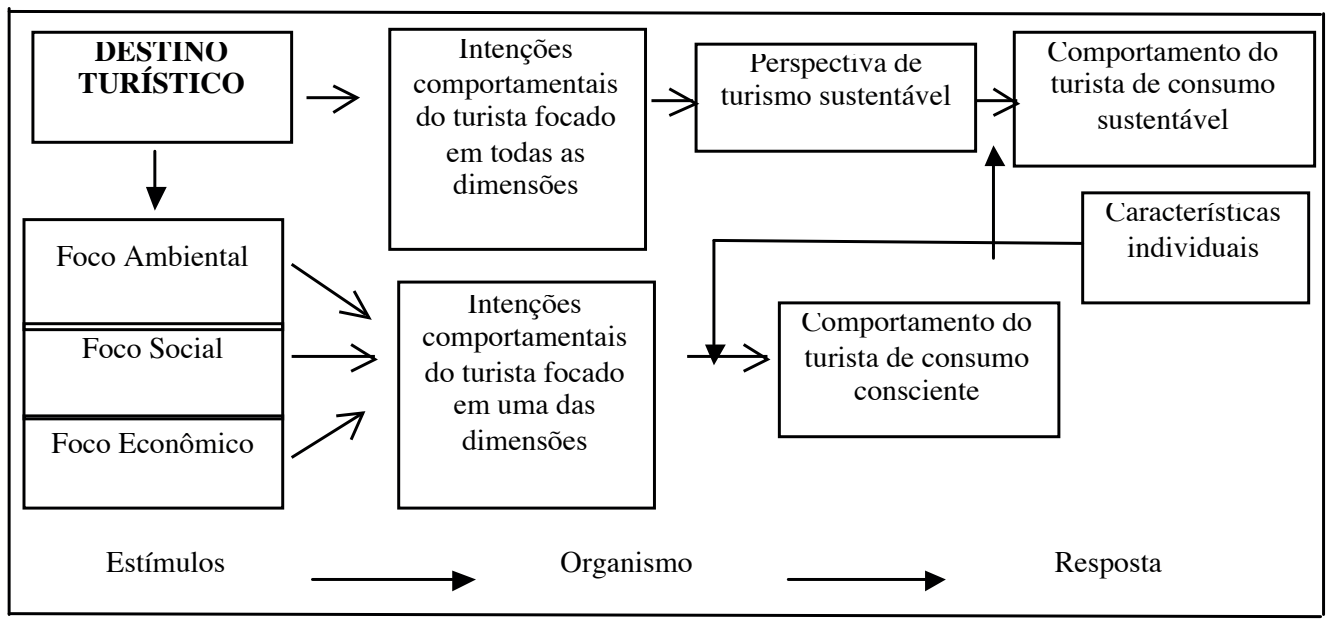

Figura 2: Perspectiva teórica de entendimento do comportamento do turista à luz de Mehrabian e Russell (1974) Fonte: os autores (2014). 
A partir da Figura 2, observa-se que o comportamento do turista pode ser influenciado pelas características do ambiente e pelo apelo às dimensões da sustentabilidade (estímulo), o que pode resultar em um turista (organismo) com intenções de consumo sustentável (resposta), quando o destino turístico considera as três dimensões simultaneamente, ou um turista (organismo) de intenções de consumo consciente (resposta), quando o destino turístico foca apenas uma das dimensões da sustentabilidade. Ressalta-se que o tipo de orientação de valor, que corresponde às características individuais que o turista possui, será uma variável moderadora sobre a intensidade da influência do ambiente sobre o indivíduo, de modo que as características do ambiente poderão influenciar de maneiras diferentes os turistas que visitarem o destino turístico.

\section{CONSIDERAÇÕES FINAIS}

Considerando o importante papel do turista no tocante à sustentabilidade dos destinos turísticos, a atenção deste artigo está voltada para o papel que o turista tem nesse processo. Como elo final da cadeia, se o turista não se comportar de forma sustentável, dificilmente a sustentabildiade do destino turístico será alcancada (HUNT, 2011). Nesse sentido, há uma tendência na literatura de buscar as características desse consumidor ou de identificar as condições que o levam a um comportamento sustentável.

No entanto, já que o turista não tem um comportamento estável no sentido de proceder da mesma forma em diferentes situações de consumo sustentável, torna-se difícil identificar a característica que o define enquanto turista sustentável. Ao contrário, a partir das observações apresentadas ao longo deste texto, é possível perceber que o consumidor sustentável não está personificado em um indivíduo padrão, pois o contexto exerce uma importante contribuição sobre seu comportamento e sobre sua direção - se será voltado para todas as dimensões concomitatemente (consumo sustentável) ou se será um comportamento de consumo consciente direcionado para a preservação ambiental, para a relevância social ou para a relação custo-benefício separadamente (MCDONALD et al., 2009).

É possível inferir que as dimensões da sustentabilidade se tornam critérios que embasam as decisões do indivíduo para que tenha ou não um comportamento sustentável. Quanto mais direcionado for um ambiente a determinada dimensão da sustentabilidade, mais esse turista vai ter um comportamento convergente a esse apelo (estímulos) presente no ambiente. As destinações que desenvolvem suas atividades com foco em apenas uma das três dimensões da sustentabilidade acabam por limitar seu papel na influência sobre o comportamento do turista (em termos da sustentabilidade ambiental, econômica e social).

Nesse sentido, a Psicologia Ambiental auxilia a entender a relação entre as características sustentáveis no destino e o comportamento sustentável subjacente (ou não), na medida em que apresenta o ambiente externo como uma importante fonte de estímulos sobre a tomada de decisão dos indivíduos. É preciso, no entanto, que os estímulos estejam presentes no ambiente turístico para que o turista perceba as características da sustentabilidade. Isso corresponde a afirmar que as destinações precisam desenhar a infraestrutura para que o indivíduo consiga identificar os fatores influenciadores que poderão moldar o seu comportamento. As ações no ambiente que promovam certo estímulo não apenas conduzirão o turista a um comportamento sustentável, mas também indicarão qual tipo de comportamento ele terá (POMERING; NOBLE; JOHNSON, 2011).

Apesar da importante contribuição apresentada neste ensaio, a discussão em torno do turismo sustentável e do comportamento sustentável do turista não está perto de ser finalizada. 0 tema é complexo e afetado por outros construtos. Sabendo-se que as informações são processadas 
internamente e os seus resultados dependem dos aspectos cognitivos do turista, é necessário averiguar como os fatores internos ao indivíduo se relacionam aos fatores externos (critérios da sustentabilidade) para gerar o consumo sustentável ou, até mesmo, como e quais tipos de informações presentes no destino podem levar o turista a um comportamento pró-sustentabilidade.

Como parte da perspectiva de um ensaio teórico, é interessante que ocorram pesquisas empíricas para fundamentar ou refutar as observações aqui apresentadas. Ressalta-se, portanto, que o intento não consistiu em esgotar todas as possibilidades de abordar o tema em questão, mas em estimular a reflexão e estudos empíricos posteriores preocupados com o desenvolvimento da atividade turística em caráter sustentável.

\section{REFERÊNCIAS}

ALIGLERI, L.; ALIGLERI, L. A.; KRUGLIANSKAS, I. Gestão socioambiental: responsabilidade e sustentabilidade do negócio. São Paulo: Atlas, 2009.

ALONSO, A.; OGLE, A. Tourism and hospitality small and medium enterprises and environmental sustainability. Management Research Review, v. 33, n. 8, 2010.

ALVES, M.; BASSANI, M. A Psicologia ambiental como área de investigação da interrelação pessoa-ambiente. In: IX Encontro de Pesquisadores e II Congresso de Iniciação Científica do Uni-FACEF, 2008, Franca. Anais... Franca: Uni-FACEF, 2008.

BASSANI, M. A. Psicologia Ambiental: contribuições para a educação ambiental. In: BOB, C.; GHITA, S.; SASEANU, A. A comparative territorial study on sustainable tourism: Romanian vs. Croatian case. Tourism \& Hospitality Management, Conference Proceedings. 33-46. 2010.

BOB, G.; SASEANU, A. A Comparative territorial study on sustainable tourism: Romanian vs Croatian case. Tourism \& Hospitality Management, Conference Proceedings, 2010.

BUARQUE, S. C. Construindo o desenvolvimento local sustentável: metodologia de planejamento. São Paulo: Garamond, 2008.

CANDREA, A.; ISPAS, A. Visitor management, a tool for sustainable tourism development in protected areas. Bulletin of the Transilvania University of Brasov, v. 51, n. 2, 2009.

CASTALDO, S.; PERRINI, F., MISANI, N.; TENCATI, A. The missing link between corporate social responsibility and consumer trust: the case of fair trade products. Journal of Business Ethics, v. 84, 2009.

CERNAT, L.; GOURDON, J. Is the concept of sustainable tourism sustainable? Developing the Sustainable Tourism Benchmarking Tool. MPRA, n. 4178, 2007. Disponível em: <http:// mpra.ub.uni-muenchen.de/4178/>. Acesso em: 05 jan. 2011.

CLAYTON, A. Climate change and tourism: the implications for the Caribbean. Worldwide Hospitality and Tourism Themes, v. 1, n. 3, 2009.

COCCOSSIS, $H$. Tourism and sustainability: perspective and implications. In: PRIESTLEY, G; EDWARDS, J.; COCCOSSIS, H. Sustainable Tourism? European Experiences. Wallingford: CAB International, 1996.

CURTIN, S; BUSBY, G. Sustainable destination development: the tour operator perspective. International Journal of Tourism Research, v. 1, 1999.

DAWKINS, J. Corporate responsibility: the communication challenge. Journal of Communication Manage. v. 9, n. 2, p. 108119, 2004.

DONYADIDE, A. Human resources empowerment and its role in the sustainable. 
Development in Tourism Industry, 2005. Disponível em: <http://ssrn.com/ abstract=1688209>. Acesso em: 25 abr. 2012

ELIGH, J.; WELFORD, R.; YTTERHUS, B. The production of sustainable tourism: concepts and examples from Norway. Sustainable Development, v. 10, n. 4, 2002.

ELKINGTON, J. Canibais com garfo e faca. São Paulo: Makroon Books, 1999.

FERGUS, A.; ROWNEY, J. Sustainable development: lost meaning and opportunity? Journal of Business Ethics, v. 60, p. 17-27, 2005.

FOLADORI, G. Por uma sustentabilidad alternativa. Uruguai: Colección Cabichui, 2005.

FREIRE, J. C.; VIEIRA, E. Uma escuta ética de psicologia ambiental. Psicologia \& Sociedade, v. 18, n. 2, p. 32-37, 2006.

GADENNE, D. L.; KENNEDY, J.; MCKEIVER, C. An empirical study of environmental awareness and practices in SMEs. Journal of Business Ethics, v. 84, p. 45-63, 2009.

GALLARZA, M.; GARCÍA, H.; SAURA, I. Destination image: towards a conceptual framework. Annals of Tourism Research, v. 29, n 1, p. 56-78, 2002.

GIFFORD, R. Environmental psychology: principles and practice. 3. ed. Boston: Optimal Books, 2002.

GIULIANI, M. V. Proposta de resposta a partir da comparação entre "Environment and Behavior" e "Journal of Environmental Psychology". Psicologia USP, v. 16, n. 1, p. 89102, 2005.

GÖSSLING, S.; BORGSTROM-HANSSON. C.; HORSTMEIER, O.; SAGGEL, S. Ecological footprint analysis as a tool to assess tourism sustainability. Ecological Economics, v.43, n.23, p.199-211, 2002.

GRAČAN, D.; SANDER, I.; RUDANČIĆ-LUGARIĆ,
A. Green strategy of business tourism. Tourism \& Hospitality Management. Conference Proceedings, 2010.

GREAVES, M.; ZIBARRAS, L.; STRIDE, C. Using the theory of planned behavior to explore environmental behavioral intentions in the workplace. Journal of Environmental Psychology, v. 34, 2013.

HARRIS, J. Basic principles of sustainable development. Global Development and Environment Institute, 2000.

HART, S. O capitalismo na encruzilhada. Porto Alegre: Bookman, 2006.

HAZIN, A.; OLIVEIRA, C.; MEDEIROS, R. Cultura e turismo: interação ou dominação? In: Trabalhos para discussão, n. 121. Outubro/2001.

HUNT, S. Sustainable marketing, equity and economic growth: a resource-advantage, economic freedom approach. Journal of the Academic Marketing Science, v. 39, p. 7-20, 2011.

HUNTER, C. Sustainable tourism as an adaptive paradigm. Annals of Tourism Research, v. 24, n. 4, p. 850-867, 1997.

JAKOBSSON, A.; MAKITALO, A.; SALJO, R. Conceptions of knowledge in research on students' understanding of the greenhouse effect: methodological positions and their consequences for representations of knowing. Science Education. v. 93, n. 6, p. 978-995, 2009.

JAMROZY, U. Marketing of tourism: a paradigm shift toward sustainability. Tourism and Hospitality Research, v. 1, n. 2, p. 117-130, 2007.

KAZAZIAN, T. Haverá a idade das coisas leves: design e desenvolvimento sustentável. São Paulo: SENAC São Paulo, 2005.

KO, T. Development of tourism sustainability 
assessment procedure: a conceptual approach. Tourism Management, v. 26, n. 3, 2005.

KÖRÖSSY, N. Do "turismo predatório" ao "turismo sustentável": uma revisão sobre a origem e a consolidação do discurso da sustentabilidade na atividade turística. Caderno Virtual de Turismo, v. 8, n. 2, 2008.

KOTLER, P.; KARTAJAYA, H.; SETIAWAN, I. Marketing 3.0: as forças que estão definindo o novo marketing centrado no ser humano. Rio de Janeiro: Elsevier, 2010.

LAKHANI, M. The need for clean production and product re-design. Journal of Cleaner Production, v. 15, 2007.

LE, Y. Perceptions of Vietnamese Tourism Businesses toward the Adoption of Sustainable Tourism Practices. Degree of doctor of philosophy with a Major in Natural Resources, 2005.

LIU, Z. Sustainable tourism development: a critique. Journal of Sustainable Tourism, v. 11, n. 6, 2003.

MACHADO, F.; PINHEIRO, C. Responsabilidade social e governança: o debate e as implicações. São Paulo: Pioneira Thomson Learning, 2006.

MARIN, L.; RUIZ, S.; RUBIO, A. The role of identity salience in the effects of corporate social responsibility on consumer behavior. Journal of Business Ethics, v. 84, p. 65-78, 2009.

MARTINS, E. C. Oturismo como uma alternativa de desenvolvimento sustentável: o caso de Jericoacoara no Ceará. Tese (doutorado em ciências) - Escola Superior de Agricultura "Luiz de Queiroz", Universidade de São Paulo, 2002.

MCDONALD, S.; OATES, C.; THYNE, M.; ALEVIZOU, P.; MCMORLAND, L. Comparing sustainable consumption patterns across product sectors. International Journal of Consumer Studies, v. 33, p. 137-145, 2009.
MCNEILL, K.; VAUGHN, M. Urban high school students' critical science agency: conceptual understandings and environmental actions around climate change. Research Science Education, v. 42, p. 373-399, 2012.

MEHRABIAN, A.; RUSSELL, J. An approach to environmental psychology. Cambridge: MIT Press, 1974.

MOSCARDO, G. Analyzing the role of festivals and events in regional development. Event Management, v. 11, 22-23, 2007.

MOSER, G. Environmental psychology and people-environment studies: what kind of multidisciplinary collaboration? Psicologia USP, v.16, n.1-2, p.131-140, 2005.

NASCIMENTO, L. Gestão socioambiental estratégica: a percepção de executivos de pequenas e médias empresas americanas. In: Encontro da Associação Nacional de PósGraduação e Pesquisa em Administração - ENANPAD, 2005, Brasília. Anais...Brasília: ENANPAD, 2005.

ORGANIZAÇÃO MUNDIAL DO TURISMO. Barômetro Mundial do Turismo, v. 8, n. 1, OMT, 2010.

ORSATO, R. Posicionamento ambiental estratégico: identificando quando vale a pena investir no verde. In: REAd, v. 30, n. 6, p. 11-46, 2006.

PAYNE, A.; STORBACKA, K.; FROW, P.; KNOX, S. Co-creating brands: diagnosing and designing the relationship experience. Journal of Business Research, v. 62, 2009.

PEATTIE, K.; COlLINS, A. Guest editorial: perspectives on sustainable consumption. International Journal of Consumer Studies, v. 33, p. 107-112, 2009.

PHIPPS, M.; OZANNE, L.; LUCHS, M.; SUBRAHMANYAN, S.; KAPITAN, S.; CATLIN, J.; GAU, R.; NAYLO, R.; ROSE, R.; SIMPSON, B.; WEAVER, T. Understanding the inherent 
complexity of sustainable consumption: a social cognitive framework. Journal of Business Research, 2012.

PINHEIRO, J. Q. Psicologia ambiental: a busca de um ambiente melhor. Estudo de Psicologia, v. 2, n. 2, p. 377-398, 1997.

POMERING, A.; NOBLE, G.; JOHNSON, L. Conceptualising a contemporary marketing mix for sustainable tourism. Journal of Sustainable Tourism, v. 19, n. 8, p. 953-969, 2011.

PORTER, M.; VAN DER LINDE, C. Verde e competitivo. In: Competição: estratégias competitivas essenciais. Rio de Janeiro: Campus, p. 371-397, 2003.

PRAHALAD, C. K. A Riqueza na base da pirâmide: como erradicar a pobreza com o lucro. Porto Alegre: Bookman, 2005.

PREBENSEN, N.; FOSS, L. Coping and cocreating in tourism experiences. International Journal of Tourism Research, p. 54-67, 2011.

RICHINS, H. Environmental, cultural, economic and socio-community sustainability: a framework for sustainable tourism in resort destinations. Environmental Development Sustainable, 2009.

ROKKA, J.; MOISANDER, J. Environmental dialogue in online communities: negotiating ecological citizenship among global travellers. International Journal of Consumer Studies, v. 33, p. 199-205, 2009.

RUSCHEINSKY, A. No conflito das interpretações: o enredo da Sustentabilidade. Revista Eletrônica do Mestrado em Educação Ambiental, v. 10, 2003.

SACHS, I. Rumo à ecossocioeconomia: teoria e prática do desenvolvimento. São Paulo: Cortez, 2007.

SANTOS, J. G. Sistema de Indicadores de Sustentabilidade para o Turismo: aplicação de uma abordagem participativa em Porto de Galinhas, PE. Dissertação de Mestrado. Programa de Pós-graduação em Administração (PROPAD/UFPE), 2013.

STEG, L.; VLEK, C. Encouraging proenvironmental behaviour: an integrative review and research agenda. Journal of Environmental Psychology, v. 29, 2009.

TOVAR, C.; LOCKWOOD, M. Social impacts of tourism: an Australian regional case study. International Journal of Tourism Research, v. I, n. 10, p. 365-378, 2008.

VAN DER ZWAN, F.; BHAMRA, T. Alternative function fulfillment: incorporating environmental considerations into increased design space. Journal of Cleaner Production, n. 11, p. 897-903, 2003.

XIANG, L.; PETRICK, J. F. Tourism marketing in an era of paradigm shift. Journal of Travel Research, v. 46, 2009.

WORLD TOURISM ORGANIZATION. Guide for Local Authorities on Developing Sustainable Tourism. Madri: WTO, 2003.

WORLDTOURISM ORGANIZATION (WTO). 2004. Disponível em: <http://www.worldtourism. org/frameset/frame_sustainable.html>. Acesso em: 04 jun. 2010. 